\title{
Avaliação da extensão dos horizontes de integração temporal do WRF nos prognósticos do vento
}

\author{
Evaluation of time range forecasting of surface wind of WRF
}

\author{
João Luiz Martins Basso*1, Luana Ribeiro Macedo ${ }^{2}$, Yoshihiro Yamasaki ${ }^{3}$ \\ ${ }^{1,3}$ Departamento da Faculdade de Meteorologia, UFPEL \\ ${ }^{2}$ Centro Estadual de Pesquisas em Sensoriamento Remoto e Meteorologia, CEPSRM
}

\begin{abstract}
Resumo
O modelo de mesoescala WRF foi configurado e processado com um domínio sobre os estado do Rio Grande do Sul utilizando como condições iniciais e de contorno os prognósticos numéricos diários do GFS (00Z, 06Z, 12Z e 18Z) durante o período de 18 a 21 de outubro de 2013. As 16 integrações foram feitas gerando prognósticos horários durantes 120 horas. A avaliação dos prognósticos dos ventos foi feita comparando os resultados com os valores registrados na estação remota de Passo Fundo - RS do INMET. O principal foco foi estabelecido sobre o dia 21 de outubro, dada a ocorrência de ventos intensos sobre a região norte do RS, especialmente em Passo Fundo. Os resultados obtidos revelam que, de uma forma geral, o WRF apresenta boa destreza nos prognósticos de ventos intensos, até mesmo com 4 dias de antecedência.
\end{abstract}

Palavras-chave: WRF, Passo Fundo, INME.

\begin{abstract}
The WRF mesoscale model has been configured and processed with a domain covering the Rio Grande do Sul state, using the GFS/NCEP forecasting (of 00Z, 06Z, 12Z and 18Z) during the October 18th to 21st, 2013 time period as initial and lateral boundary conditions. The 16 model integrations have been processed, generating hourly forecasting up to 120 hours. The surface wind forecasting have been evaluated throughout comparison with the data as collected by the INMET remote station installed at Passo Fundo city - RS. The main focus has been established on October 21

st, due the occurrence of quite intense winds over the whole northern sector of Rio Grande do Sul state, particularly over Passo Fundo city. The gathered results reveal that, in a general sense, the WRF presented very good skill for strong wind, as well as - even up to ninety six hours ahead.
\end{abstract}

Keywords: WRF, Passo Fundo, INMET. 


\section{Introdução}

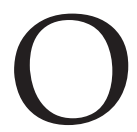

WRF (Weather Research and Forecasting) constitui um complexo sistema de modelagem de mesoescala de previsão numérica do tempo da atmosfera. Ele vem sendo crescentemente utilizado, tanto para fins de pesquisa como também para as atividades operacionais pelos centros de previsão de tempo (Skamarock et al. 2008). Esse sistema de modelagem vem sendo desenvolvido com o suporte e esforço coletivo, de pesquisadores de vários centros de pesquisa e organizações como o National Center for Atmospheric Research (NCAR); o National Oceanic and Atmospheric Administration (NOAA); o National Centers for Environmental Prediction (NCEP); o Forecast System Laboratorory (FSL); o Department of Defense - Air Force Weather Agency (AFWA) e Naval Research Laboratory (NRL); o Center for Analysis and Prediction of Storms (CAPS); a Federal Aviation Administration (FAA); além de colaboradores internacionais de diversas instituições.

O sistema WRF apresenta um conjunto avançado da modelagem atmosférica e conta com dois núcleos dinâmicos (MMM e ARW). Apresenta, além disso, módulos que constituem o estado da arte dos desenvolvimentos científicos e tecnológicos dos complexos sistemas de assimilação de dados 3DVAR e 4DVAR. No tocante às aplicações ambientais, ele permite a exploração de uma ampla gama de fenômenos meteorológicos, com escalas espaciais que vão desde metros até milhares de quilômetros. Ele permite, dentre outros, a realização de simulações numéricas com dados reais, provenientes de observações ou análises, ou de condições atmosféricas idealizadas.

Visto que o WRF integra um sistema de equações altamente não lineares de previsão de tempo, haverá sempre erros, ou desvios, em relação a parâmetros atmosféricos observados. Em particular esse fato ocorre ao estender o horizonte de integração numérica no tempo. É importante ressaltar que, pelo fato do modelo numérico proceder a integração temporal, a partir de um estado inicial conhecido e fornecido - que no caso do WRF de área limitada - é feito empregando-se dados de modelos globais - pode induzir a erros nas previsões, face aos erros inerentes aos instantes iniciais da integração numérica. Além disso, há também e dentre outros, a incapacidade, em especial, a computacional - de incorporar todas as fenomenologias termodinâmicas da atmosfera, com suas escalas de variações temporais e espaciais - que podem produzir erros nas previsões.

O estudo foi conduzido com o objetivo de explorar os efeitos da extensão do horizonte da integração temporal, com o processamento do modelo WRF sobre o estado do Rio Grande do Sul. Serão apresentados apenas os resultados das análises dos diferentes prognósticos, feitos com o processamento do modelo com distintas condições iniciais e de fronteira, para a magnitude do vento a 10 metros da superfície.

\section{Materiais e Métodos}

\subsection{Configuração do Modelo WRF}

O modelo WRF, constituído pelas equações não hidrostáticas, foi configurado com um domínio de integração englobando o estado do Rio Grande do Sul, conforme apresentado na Figura 1.1. Esse domínio de integração apresenta 63 pontos de grade na direção leste-oeste e 58 pontos de grade na direção norte-sul e 35 níveis $\eta$ (eta) na vertical - com topo em $50 \mathrm{hPa}$. Esses pontos de grade foram definidos para cada $18 \mathrm{~km}$ de distância entre eles e, portanto, modelados com resolução espacial de $36 \mathrm{~km}$ nas 16 simulações numéricas. Foram realizadas processando o modelo WRF com horizonte de integração de 120 horas e armazenamento dos prognósticos hora em hora. Cada um dos quatro prognósticos numéricos do GFS - produzidos pela NCEP - às 00:00Z, 06:00Z, 12:00Z e 18:00Z nos dias 18 a 21 de outubro de 2013, foram utilizados como condições iniciais e de fronteira na a integração numérica do modelo WRF. Como resultado das simulações do WRF, os processamentos constituíram um total de 16 arquivos prognósticos

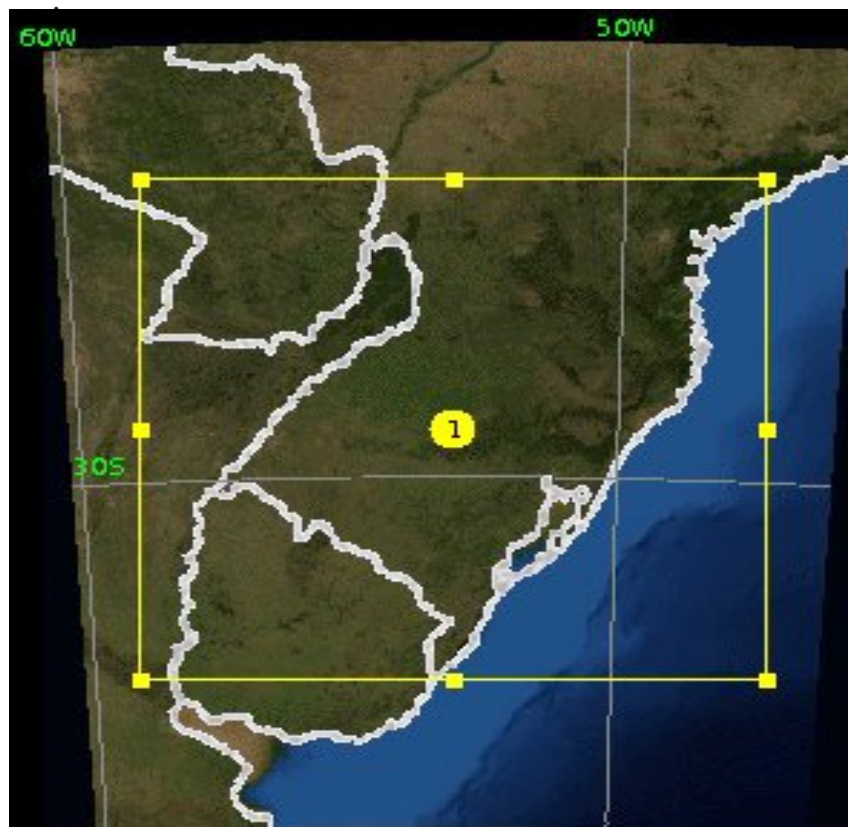

Figura 1.1: Domínio de integração do modelo WRF.

\subsection{Processamento do Modelo de Mesoescala}

O processamento do modelo WRF foi realizado utilizando, como condições iniciais e de contorno, os prognósticos numéricos, no formato GRIB2, do modelo global GFS do NCEP com resolução espacial de $0,5^{\circ} \mathrm{e}$ configurado com as parametrizações conforme apresentadas na Tabela 1.

Ressalta-se que o desempenho do modelo de previsão WRF, assim como todos os modelos de previsão 
de tempo, apresenta uma grande dependência, dentre outras, das condições iniciais utilizadas. Por conseguinte é importante dispor de dados que representam o estado inicial da atmosfera, mais próximo da realidade quanto possível. Essa abordagem será apresentada como continuidade da presente análise preliminar. A avaliação sobre a extensão temporal do horizonte de integração numérica, sobre os prognósticos numéricos do WRF é feita mediante a comparação, entre os valores previstos pelo modelo e os que foram registrados na estação remota A839 de Passo Fundo RS, do Instituto Nacional de Meteorologia (INMET), instalada nas coordenadas $28,2294^{\circ} \mathrm{S}$ e $52,4039^{\circ} \mathrm{O}$.

Tabela 1: Parametrizações utilizadas no modelo de mesoescala WRF.

\begin{tabular}{|l|l|}
\hline Parametrizações & Domínio \\
\hline Microfísica & Thompson \\
\hline Radiação OL & RRTMG \\
\hline Radiação OC & RRTMG \\
\hline CLP & Mellor-Yamada -Janjic \\
\hline Cumulus & Kain-Fritsch \\
\hline
\end{tabular}

\section{Resultados}

A estação do INMET registrou no dia 21 de outubro de 2013 , ventos e rajadas de vento de $6,2 \mathrm{~m} / \mathrm{s}$ e $10,5 \mathrm{~m} / \mathrm{s}$, às $08 \mathrm{UTC}$ e de $5,9 \mathrm{~m} / \mathrm{s}$ e $9,9 \mathrm{~m} / \mathrm{s}$ às 09 UTC respectivamente. Durante o período da madrugada, entre 03 UTC e até às 11 UTC da manhã, as intensidades de vento registradas pela estação do INMET, permaneceram acima de $5 \mathrm{~m} / \mathrm{s}$ $(18 \mathrm{~km} / \mathrm{h})$.

O período em que foi dada ênfase no estudo, dia 21 de outubro de 2013, foi o analisado, entre as 08 e 09 UTC - quando houve ventos intensos; que, segundo relatos do jornal O Nacional, destelharam prédios e derrubaram árvores na cidade.

Os campos de intensidade de ventos, meteogramas da intensidade dos ventos mostrando a variação da intensidade de intensidade dos ventos observados pela estação remota do INMET, pelas simulações realizadas serão apresentados na sequência. Serão também apresentados os desvios das simulações realizadas.

\subsection{Campos de intensidade de vento}

Os campos de intensidade de vento, gerados a partir do modelo de mesoescala que serão apresentados, comparando-se com os dados de velocidade de vento registrados pela estação do INMET, são provenientes das previsões do WRF, geradas a partir dos prognósticos das 00Z, do dia 19 de outubro de 2013 e das 06Z do dia 20 de outubro de 2013.

No prognóstico das 08 UTC, processado com inicia- lização às $00 Z$ do dia 19 de outubro, apresentado nas Figuras 1.2 e 1.3, foi previsto um vento com intensidade entre 6 e $7 \mathrm{~m} / \mathrm{s}$ e uma hora após, intensidade entre 5 e 6 $\mathrm{m} / \mathrm{s}$. Por outro lado, o prognóstico das 08 UTC processado, com inicialização às 06 UTC do dia 20 de outubro apresentaram intensidade de vento entre 6 e $7 \mathrm{~m} / \mathrm{s}$ entre 4 e 6 m/s uma hora depois, conforme as Figuras 1.4 e 1.5.

Os campos de previsão de vento gerados a partir dos prognósticos inicializados às 00Z, 06Z 12Z e 18Z do dia 18 de outubro de 2013 apresentaram, às 08 UTC intensidade de vento variando entre 4 e $5 \mathrm{~m} / \mathrm{s}$ e entre 4 e $6 \mathrm{~m} / \mathrm{s}$, às 09 UTC. Esses resultados são consideráveis, visto que são de previsões de até $80 \mathrm{~h}$ antes da ocorrência e registro da estação meteorológica.

Os campos da previsão de vento gerados a partir dos prognósticos do dia 19 de outubro de 2013 inicializando às 06Z, 12Z e 18Z apresentam desvios significativos comparados entre si. Os resultados das 06Z, por exemplo, apresentam ventos com intensidade entre 5 e $6 \mathrm{~m} / \mathrm{s}$, às 08 UTC e entre 4 e $5 \mathrm{~m} / \mathrm{s}$ às 09 UTC. Já os resultados das $12 \mathrm{Z}$ e $18 \mathrm{Z}$ apresentam ventos de intensidade entre 7 e 8 $\mathrm{m} / \mathrm{s} ; 8$ e $9 \mathrm{~m} / \mathrm{s}$ às 08 UTC; entre 4 e $5 \mathrm{~m} / \mathrm{s}$ e 6 e $8 \mathrm{~m} / \mathrm{s}$, às 09 UTC, respectivamente.

Os resultados gerados a partir do processamento dos prognósticos do dia 20 de outubro de 2013 das 00Z, $12 Z$ e $18 Z$ apresentaram ventos entre 8 e $10 \mathrm{~m} / \mathrm{s}$, às 08 UTC e entre 8 e 12 m/s, às 09 UTC. Resultados que aproximam da velocidade de rajadas de vento registradas pela estação meteorológica do INMET.

As previsões de vento geradas a partir de prognósticos do dia 21 de outubro de 2013 das 00Z apresentaram ventos de intensidade entre 8 e $10 \mathrm{~m} / \mathrm{s}$ às 08 UTC e entre 12 e 14 $\mathrm{m} / \mathrm{s}$ às 09 UTC. Resultados próximos do registrado de rajadas de vento das 08 e 09 UTC. Os campos gerados a partir do prognóstico das $06 Z$ do dia 21 de outubro de 2013 apresentaram vento de intensidade entre 4 e 6 $\mathrm{m} / \mathrm{s}$ às 08 UTC e entre 5 e 6 m/s às 09 UTC.

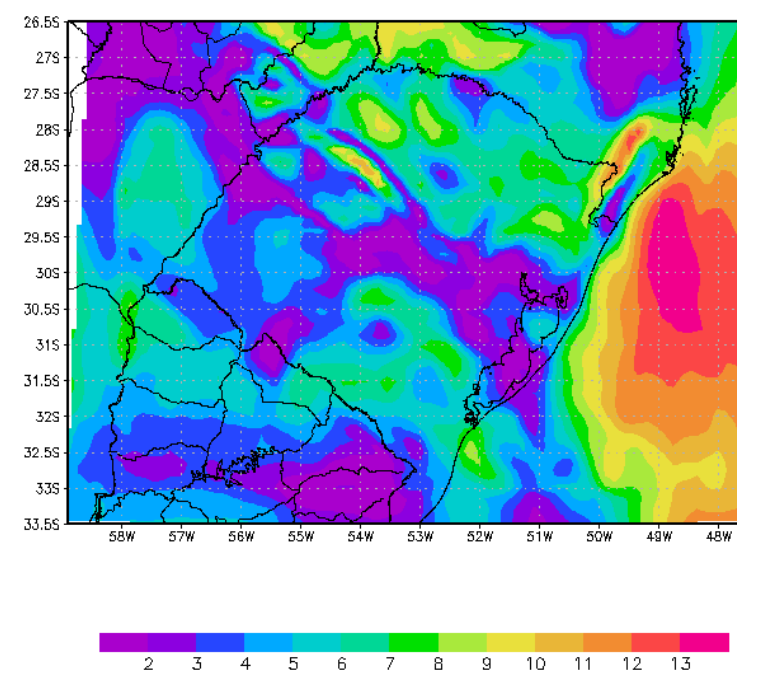

Figura 1.2: Intensidade do vento prevista para as 08 UTC com inicialização em 19/10/2013 - 00UTC. 
Magnitude do Vento $10 \mathrm{~m}$ ogz220cr2013

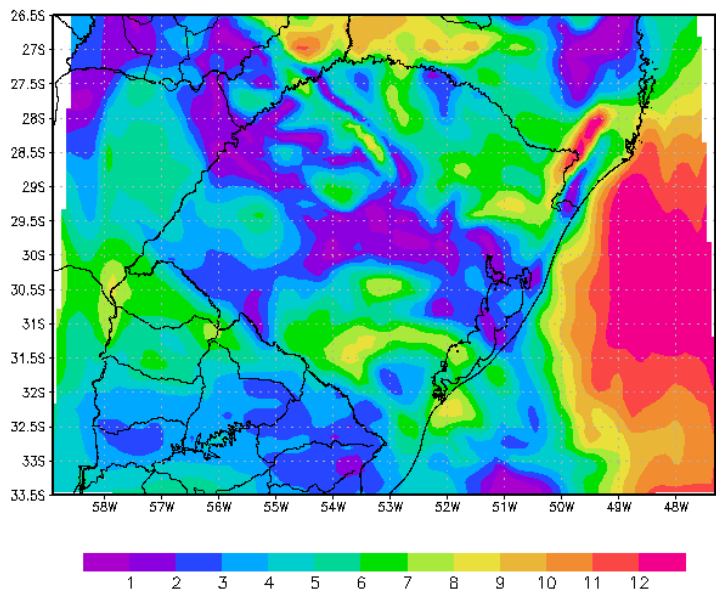

Figura 1.3: Intensidade do vento prevista para as 09 UTC com inicialização em 19/10/2013 - 00UTC.

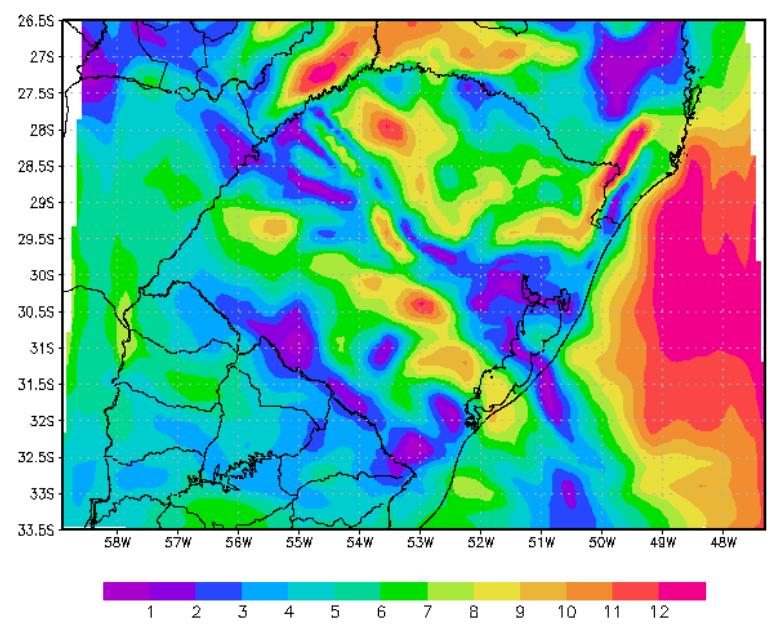

Figura 1.4: Intensidade do vento prevista para as 09 UTC com inicialização em 20/10/2013 - 06UTC.

Magnitude do Vento $10 \mathrm{~m}$ ogz210072013

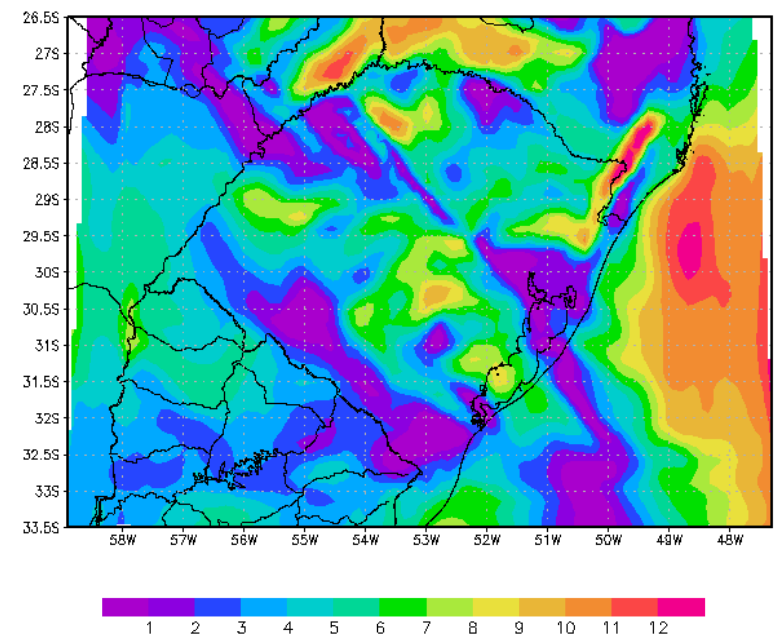

Figura 1.5: Intensidade do vento prevista para as 09 UTC com inicialização em 20/10/2013 - 06UTC.

\subsection{Meteogramas e Desvios}

Centros de Pesquisa e de Previsões meteorológicas frequentemente apresentam meteogramas para representar a variação temporal das variáveis de interesse. Nos casos de simulações atmosféricas, realizadas com modelos meteorológicos, é interessante apresentar os desvios dos dados simulados com relação aos dados observados. Estas diferenças são apresentadas nas Figuras 3.1, 3.2, 3.3 e 3.4.

Os meteogramas que são apresentados nas Figuras 2.1, 2.2, 2.3 e 2.4 foram gerados a partir das saídas das simulações de vento.

Os prognósticos numéricos, nos instantes iniciais de integração, de até cerca de 2 horas eventualmente não representam as características reais da atmosfera, por que o modelo de mesoescala possui um tempo de spin-up - período em que o modelo ajusta as condições iniciais ao sistema de equações altamente não lineares do modelo. É possível inferir que os dados observados para o dia 18 de outubro são representados com precisão em certos períodos de tempo.

O meteograma apresentado na Figura 2.1, apresenta as simulações realizadas a partir do dia 18 de outubro de 2013 e apresenta juntamente com os valores observados do vento.

Pode-se observar, nas Fig. 2.1 e 3.1, que as intensidades de ventos simulados a partir do prognóstico das 00Z, das 06Z e das $12 Z$ apresentam a variação da velocidade do vento observada com pequenos desvios durante o período das 12 até próximo às 20 horas. A partir das $18 \mathrm{~h}$ de integração, quando foi inicializada a simulação do WRF das 18Z. Observa-se que, posterior ao início do último prognóstico e até cerca de $22 \mathrm{~h}$ de integração, todas as simulações realizadas acompanham com pequena superestimação com relação aos valores observados.

O período que compreendido entre 22 horas próximo das 28 horas de integração, há grandes variações nas velocidade dos ventos de dados observados, caracterizando de forma bem clara se tratar de rajadas de vento. Características como esta são particularidades de difícil simulação pelo modelo, quando se extende o horizonte temporal da simulação. Esse fato pode ser inferido observando as Figuras 2.1 e 3.1, onde a melhor representatividade no período é apresentada pela simulação do prognóstico das $18 Z$.

Após as $28 \mathrm{~h}$ de integração numérica, até próximo de 35 horas, observa-se que as simulações realizadas a partir dos prognósticos das 00Z e 06Z apresentam com maior precisão a variação da intensidade dos ventos. Porém das 38 até 40h de integração, a melhor representação é apresentada pela simulação do prognóstico das $12 Z$. Posterior às $40 \mathrm{~h}$ e até próximo de $52 \mathrm{~h}$ de integração, as simulações representam com precisão a comparado com os valores observados, salvo a pequenos desvios que podem ser observados, tanto na Fig. 2.1, como na Fig. 3.1. 


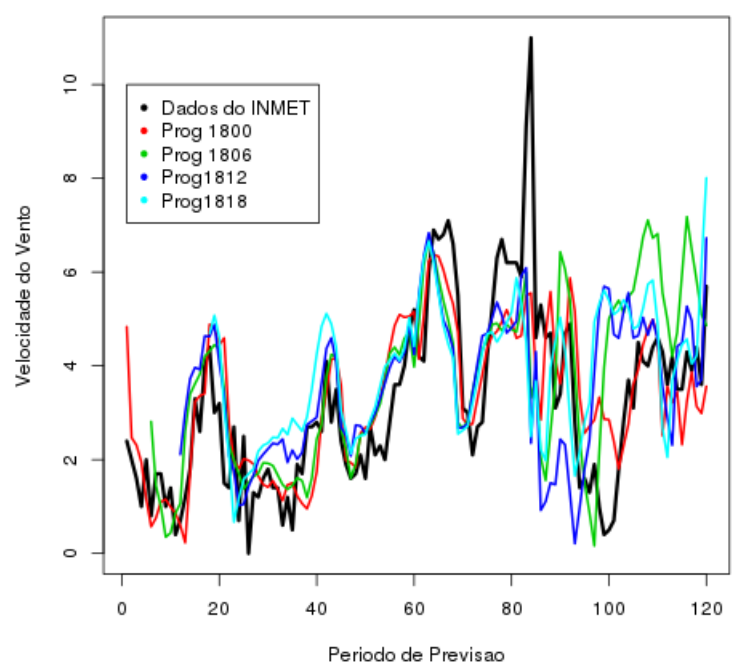

Figura 2.1: Velocidade dos ventos observados e simulados com horizonte de previsão para $120 \mathrm{~h}$.

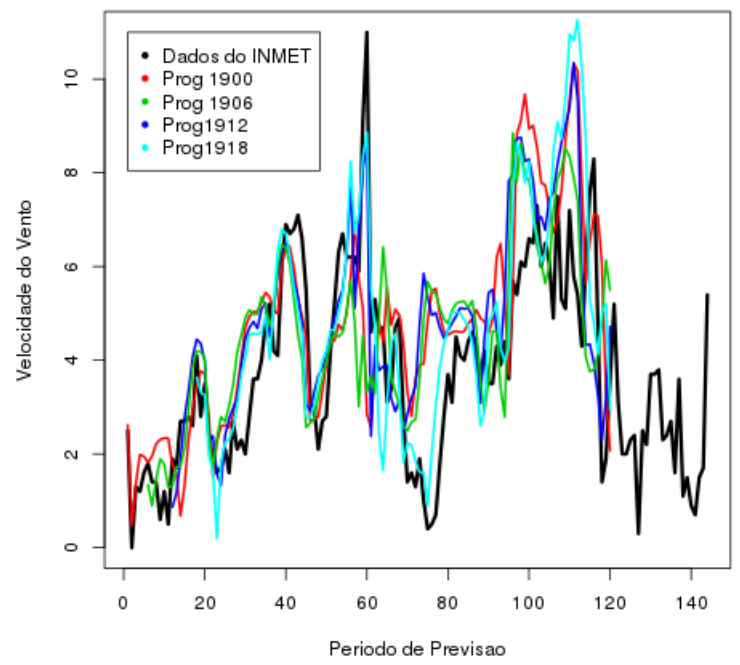

Figura 2.2: Velocidade dos ventos observados e simulados com horizonte de previsão para $120 \mathrm{~h}$.

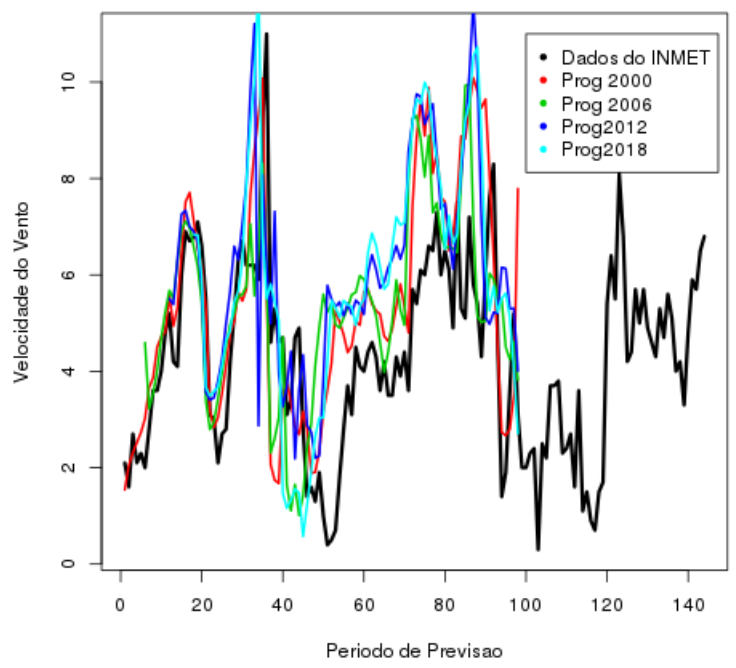

Figura 2.3: Velocidade dos ventos observados e simulados com horizonte de previsão para 120h.

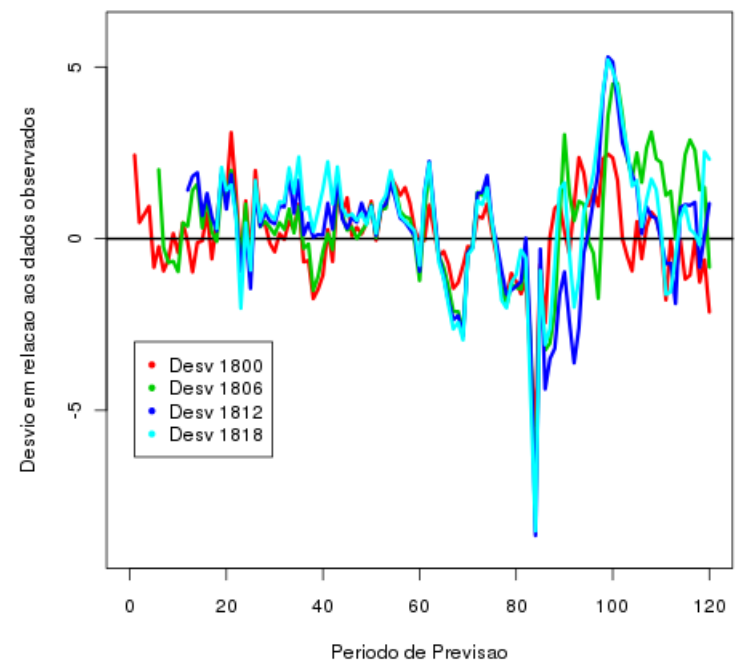

Figura 3.1: Desvio dos dados simulados com horizonte de previsão para $120 \mathrm{~h}$.

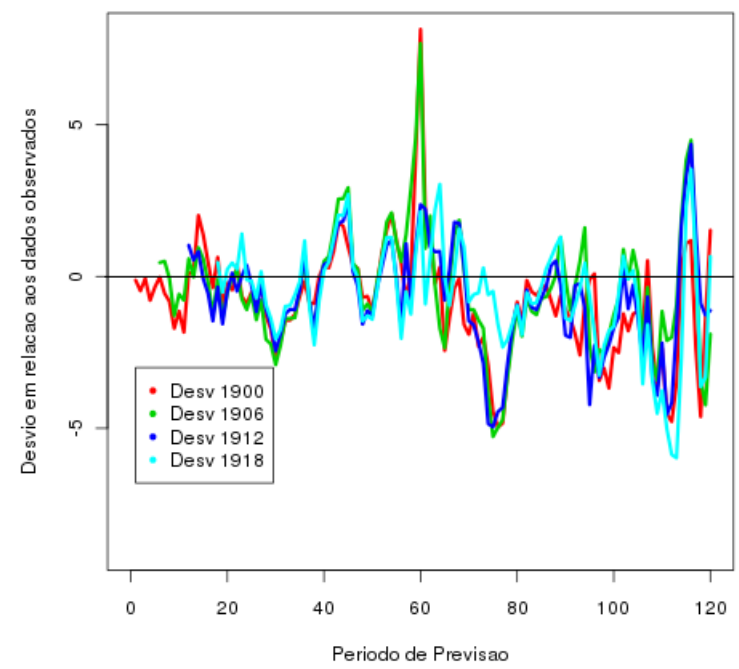

Figura 3.2: Desvio dos dados simulados com horizonte de previsão para $120 \mathrm{~h}$.

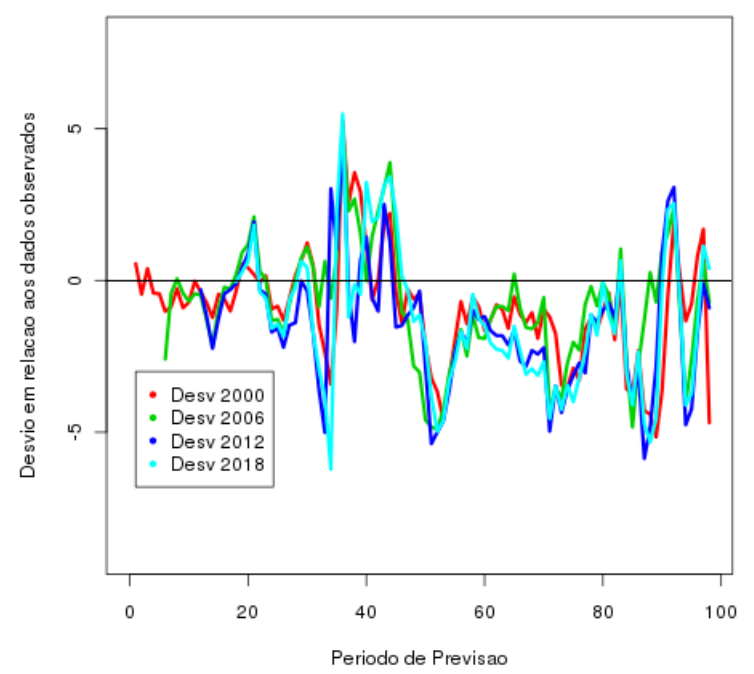

Figura 3.3: Desvio dos dados simulados com horizonte de previsão para $120 \mathrm{~h}$. 
Pode-se observar que nas $52 \mathrm{~h}$ posteriores ao início da observação até $58 \mathrm{~h}$ e das $61 \mathrm{~h}$ até próximo das $63 \mathrm{~h}$, o modelo superestima a intensidade dos ventos em até 2 $\mathrm{m} / \mathrm{s}$ e dentro desse intervalo subestima em até $1 \mathrm{~m} / \mathrm{s}$ em todas as simulações realizadas. A partir das $63 \mathrm{~h}$, pode-se observar que a simulação realizada a partir do prognóstico das $00 Z$ é a que representa com maior precisão a variação sobre as observações, conforme a Fig. 3.1, onde os desvios observados são menores quando comparados às simulações realizadas, a partir dos prognósticos das 06Z, 12Z e 18Z.

Nas figuras 2.2 e 3.2, nota-se que as simulações realizadas para o dia 19 de outubro apresentam nos instantes de tempo iniciais, entre 0 e 24 horas de simulação, maior precisão na representação da intensidade dos ventos comparado com as simulações realizadas para o mesmo período de tempo do dia 18 de outubro. Relacionando essas simulações nota-se um padrão para este período de tempo.

Para o período entre 24 e próximo das 48 horas, a intensidade dos ventos simulada a partir dos prognósticos das $12 Z$ e $18 Z$ do dia 19, apresentaram resultados semelhantes às simulações realizadas a partir dos prognósticos das $12 Z$ e $18 Z$ do dia 18. Entretanto durante um curto intervalo próximo das $40 \mathrm{~h}$ de simulação - a intensidade simulada advinda do prognóstico das $12 Z$ do dia 18, apresentou erros não significativos em relação aos dados observados.

Assim como nas simulações realizadas para o dia 18, a intensidade do vento para o período em que o vento apresenta grandes variações e com características de rajadas notam-se dificuldades nas simulações do modelo de mesoescala WRF. É possível visualizar isto quando a previsão se aproxima das $60 \mathrm{~h}$ do dia 19. Porém as simulações realizadas a partir dos prognósticos das 12 e 18Z, do mesmo dia, acompanham esta variação (não perfeitamente) - mas possibilitando concluir que prognósticos de condições iniciais mais próximas da ocorrência de certos eventos, neste caso, a de rajadas - tendem a acompanhar de forma mais precisa os dados obtidos pelo modelo, para com os dados reais observados. Pode-se observar, por exemplo, que no período entre $65 \mathrm{~h}$ e até próximo das $90 \mathrm{~h}$ de previsão, a simulação realizada, que menos apresenta erros, é feita a partir do prognóstico das $18 Z$ do dia 19.

Posterior às $100 \mathrm{~h}$ de previsão, o modelo dificilmente aproxima às condições atmosféricas reais com precisão. Apresenta os desvios nas simulações realizadas em boa parte dos prognósticos.

As simulações realizadas a partir dos prognósticos do dia 20 foram realizadas para um período que acompanhasse o evento ocorrido no dia 21. Para os períodos observados nas figuras 2.3 e 3.3, pode-se notar que para os períodos iniciais, entre 0 e $24 \mathrm{~h}$ de previsão - acompanhando a intensidade dos ventos do dia 20 - o modelo, assim como nas simulações realizadas e apresentadas nos gráficos anteriores (Figuras 2.1, 2.2, 3.1 e 3.2) aproximam a intensidade dos ventos com desvios relativamente pequenos. No entanto quando as simulações aproximam da previsão de 24 até $48 \mathrm{~h}$, as rajadas de vento apresentadas nos dados observados são nítidas, e como descrito anteriormente, dificultam a previsão realizada a partir do modelo.

Simular a intensidade dos ventos para o dia 21, dia em que foram registradas altas intensidades e de grande variação do vento registrados pela estação de dados do INMET foi tentativa de várias previsões. Entretanto, pode-se observar que o modelo apresenta dificuldades na caracterização das grandes variações do vento em curto intervalo de tempo (rajadas).

\section{Conclusões}

Os resultados gerados pelo modelo de mesoescala WRF responderam muito bem, principalmente para as intensidades de vento previstos para os períodos iniciais do horizonte de integração. Isto se deve ao fato de terem apresentado desvios bem pequenos nos períodos iniciais de integração sobre o domínio do modelo com alta resolução espacial. Ao extender o horizonte de integração, as simulações realizadas respondem com menor precisão, porém isto ocorreu devido às rajadas de vento, durante certos períodos de tempo, especialmente no dia 21 de outubro de 2013, quando ocorreu uma tempestade sobre a cidade de Passo Fundo. Nos instantes posteriores a esse forte evento, as simulações apresentaram desvios significativamente pequenos comparadas com os observados.

Os prognósticos e as análises revelaram que o modelo WRF apresenta resultados próximos à realidade, exceto quando ocorrem grandes variações temporais nas intensidades do vento. Além disso é importante observar que o modelo apresenta boa destreza até mesmo para relativamente longo prazo (4 dias) de previsão.

\section{Agradecimentos}

Ao INMET, pela disponibilização dos dados de estações automáticas, e a todas outras instituições que, de alguma forma contribuíram para a realização da pesquisa.

\section{Referências}

INMET disponível em: <www.inmet.gov.br>, Acesso em 23 de Outubro de 2013.

Jornal O Nacional, disponível em: <http:// www.onacional.com.br/geral/cidade/42833/ temporal+causa+estragos+em+passo+fundo $>$, Acesso em 23 de Outubro de 2013.

Skamarock, W.C.; et al., 2008: A Description of the Advanced Research WRF Version 3 . NCAR/TN 475+STR Tech. Note, UCAR, 2008. 